${ }^{2}$ Graduate School of Medicine, Kyoto University, Department of Advanced Medicine for Rheumatic Diseases, Kyoto, Japan; ${ }^{3}$ Graduate School of Medicine, Kyoto University, Center for Genomic Medicine, Kyoto, Japan; ${ }^{4}$ Center for Integrative Medical Sciences, RIKEN, Laboratory for Statistical and Translational Genetics, Yokohama, Japan; ${ }^{5}$ Shizuoka General Hospital, Clinical Research Center, Shizuoka, Japan; ${ }^{6}$ The School of Pharmaceutical Sciences, University of Shizuoka, The Department of Applied Genetics, Shizuoka, Japan

Background: We have previously identified single nucleotide polymorphism (SNP) rs6871626 in IL12B, rs103294 in LILRA3, rs17133698 in DUSP22, rs2322599 in PTK2B, and rs1713450 in KLHL33 as non-HLA susceptibility loci in Takayasu arteritis (TAK) [1, 2]. However, the association of these SNPs with clinical features has scarcely investigated.

Objectives: In this study, we aimed to examine how these SNPs contribute to clinical features and vascular damage in TAK.

Methods: We enrolled 99 TAK patients who were enrolled in our previous genome-wide association study (GWAS) [2]. To assess vascular damage, Takayasu Arteritis Damage Score (TADS) and Vasculitis Damage Index (VDI) were measured at the last visit before November 2020. As for organ damages, the presence or absence of aortic regurgitation (AR), hypertension, ischemic heart disease, cerebrovascular event, visual loss, end-stage renal failure, and inflammatory bowel disease were evaluated. Treatment profiles including immunosuppressive drugs and vascular interventions were also reviewed.

Results: The incidence of AR was positively associated with the risk allele of $I L 12 B$ rs6871626 ( $p=0.0052$; odds ratio (OR) $2.45,95 \%$ confidence interval $(\mathrm{Cl}) 1.27-4.73$ ), and so was the proportion of patients who underwent aortic valve replacement $(\mathrm{p}=0.023$; OR $3.64,95 \% \mathrm{Cl} 1.08-12.24)$ (table 1). The incidence of hypertension was associated with the risk allele of $I L 12 B$ rs $6871626(p=0.049$; OR $1.82,95 \% \mathrm{Cl}$ 0.99-3.36) and PTK2B rs2322599 ( $\mathrm{p}=0.044$; OR 2.52, 95\% Cl 0.97-6.54) (table 1). The proportion of biologic users tended to be higher in the risk genotypes of IL12B rs6871626 ( $\mathrm{p}=0.15$; OR1.80, 95\% $\mathrm{Cl}$ 0.79-3.99). Regarding vascular damage, there was positive correlation between TADS and the risk allele of IL12B rs6871626 $(p=0.0035 ; \beta=1.35)$ (Figure 1$)$. Moreover, VDI was also positively correlated with the allele $(p=0.0054 ; \beta=0.96)$ (Figure 1). No other clinicogenetic associations were observed between five SNPs and vasculitis-associated damages.

Table 1. The association of the five SNPs with aortic regurgitation and hypertension

\begin{tabular}{lllll}
\hline & Aortic regurgitation & \multicolumn{3}{l}{ Hypertension } \\
& OR $(95 \% \mathrm{Cl})$ & p value & OR $(95 \% \mathrm{Cl})$ & p value \\
\hline IL12B rs6871626 & $2.45(1.27-4.73)$ & $0.0052^{*}$ & $1.82(0.99-3.36)$ & $0.049^{*}$ \\
PTK2B rs2322599 & $1.21(0.51-2.86)$ & 0.67 & $2.51(0.97-6.54)$ & $0.044^{*}$ \\
LILRA3 rs103294 & $1.16(0.52-2.61)$ & 0.71 & $1.20(0.55-1.64)$ & 0.64 \\
DUSP22 rs17133698 & $0.56(0.28-1.13)$ & 0.090 & $0.87(0.46-1.63)$ & 0.66 \\
KLHL33 rs1713450 & $0.89(0.42-1.91)$ & 0.77 & $1.48(0.68-3.22)$ & 0.31
\end{tabular}

SNP, single nucleotide polymorphism; OR, odds ratio; $\mathrm{Cl}$, confidence interval

Figure. The association of the five SNPs with VDI
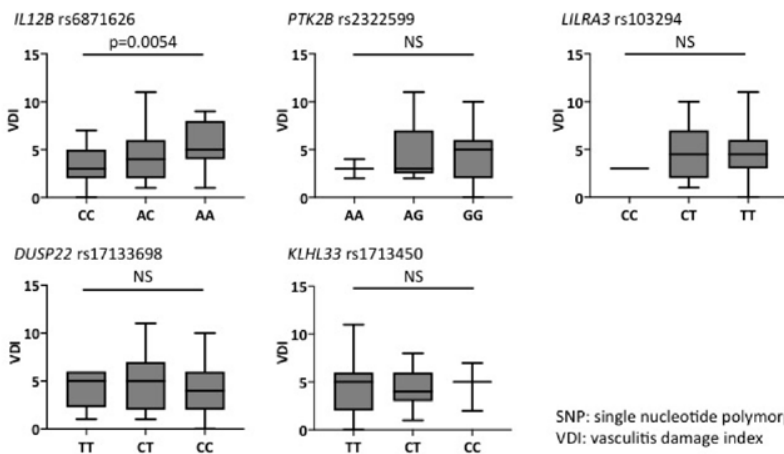

SNP: single nucleotide polymorphism

Conclusion: In the present study, IL12B rs6871626 was closely correlated with vascular damage. We also found association between PTK2B rs2322599 and hypertension. There was no significant relevance between vascular damage and LILRA3 rs103294, DUSP22 rs17133698, or KLHL33 rs1713450.

REFERENCES:

[1] Terao C et al. Am J Hum Genet. 2013;93(2):289-97.

[2] Terao C et al. Proc Natl Acad Sci U S A. 2018;115(51):13045-50.

Disclosure of Interests: Keiichiro Kadoba: None declared, Ryu Watanabe Speakers bureau: I have received speaker's fee from Mitsubishi Tanabe Pharma, Pfizer, Sanofi, AbbVie, Asahi Kasei, Eisai, Eli Lilly, Bristol-Myers Squibb, and Janssen., Takeshi Iwasaki: None declared, Koji Kitagori Grant/research support from: KK has received research grants from GlaxoSmithKline., Syuji Akizuki: None declared,
Kosaku Murakami Speakers bureau: I have received speaking fees from Eisai Co. Ltd, Chugai Pharmaceutical Co. Ltd., Pfizer Inc., Bristol-Myers Squibb, Mitsubishi Tanabe Pharma Corporation, UCB Japan Co. Ltd, Daiichi Sankyo Co. Ltd. and Astellas Pharma Inc., Ran Nakashima: None declared, Motomu Hashimoto Speakers bureau: I have received a research grant and/or speaker fee from Bristol-Myers, Eisai Ely Lilly, Mitsubishi Tanabe Pharma., Grant/research support from: I have received a research grant and/or speaker fee from Bristol-Myers, Eisai, Ely Lilly, Mitsubishi Tanabe Pharma., Masao Tanaka Speakers bureau: I have received research grants and/or speaker fees from AbbVie GK, Asahi Kasei Pharma Corporation, Astellas Pharma Inc., Bristol-Myers Squibb, Chugai Pharmaceutical Co., Ltd., Eisai Co., Ltd. Eli Lilly and Company, Pfizer Inc., UCB Japan Co., Ltd., Janssen Pharmaceutical K.K., Mitsubishi Tanabe Pharma Corporation, Novartis Pharma K.K., Taisho Pharma Co., Ltd, and Takeda Pharmaceutical Company Limited., Koichiro Ohmura Speakers bureau: I have received speaker's fee from Abbvie, Actelion, Asahikasei Pharma Astellas, AYUMI, Bristol-Myers Squibb, Chugai, Daiichi-Sankyo, Eisai, Eli Lilly, GSK Janssen, JB, Mitsubishi Tanabe, Nippon Kayaku, Nippon Shinyaku, Novartis, Sanof and Takeda., Grant/research support from: I have received research grants from GlaxoSmithKline., Akio Morinobu Speakers bureau: I have received speaking fees from Chugai Pharmaceutical Co. Ltd., Grant/research support from: I have received research grants from Chugai Pharmaceutical Co. Ltd., Chikashi Terao: None declared, Hajime Yoshifuji Speakers bureau: I have received lecture fees from Chugai., Consultant of: I have been an advisory board for a clinical trial conducted by Janssen. DOI: 10.1136/annrheumdis-2021-eular.289

\section{POS0346 THE THERAPEUTIC EFFECTS OF OMEGA-6 PUFA (PINOLENIC ACID) ON THE TRANSCRIPTOMIC PROFILE OF ACTIVATED PERIPHERAL BLOOD MONONUCLEAR CELLS ISOLATED FROM HEALTHY CONTROLS AND RHEUMATOID ARTHRITIS PATIENTS}

R. Takala ${ }^{1,2}$, D. Ramji ${ }^{2}$, R. Andrews ${ }^{1}$, Y. Zhou ${ }^{1}$, J. Burston ${ }^{1}$, E. Choy ${ }^{1,3} .{ }^{1}$ Cardiff University, Division of Infection and Immunity, School of Medicine, Cardiff, United Kingdom; ${ }^{2}$ Cardiff University, School of Biosciences, Sir Martin Evans Building, Cardiff, United Kingdom; ${ }^{3}$ Cardiff University, CREATE Centre, Section of Rheumatology, School of Medicine, Cardiff, United Kingdom

Background: Globally, Rheumatoid arthritis (RA) is the most common chronic inflammatory arthritis. EULAR recommends patient education and disease modifying anti-rheumatic drugs including advanced targeted therapies for management ${ }^{12}$. Many patients seek advice on a dietary intervention that may improve symptoms. Foods high in omega-3 polyunsaturated fatty acids (PUFAs) is one of the most common recommended based on their anti-inflammatory properties 4. Previously we showed pinolenic acid (PNLA), an omega-6 (PUFAs) found in pine nuts, reduced lipid uptake, endocytosis and migration of monocyte in vitro. PNLA also reduced IL-6, TNF- $\alpha$, IL-1 $\beta$ and PGE2 produced by lipopolysaccharide (LPS) activated PBMCs from patients with RA and healthy controls (HCs) Objectives: We hypothesis that PNLA inhibits key inflammatory processes in synovities as in diagram.1. We investigated the transcriptomic profile of PNLA treatment on LPS activated PBMCs isolated from $\mathrm{HCs}$ and RA patients.

Methods: Adult RA patients $(n=6)$ were recruited from the Rheumatology Department of University Hospital of Wales together with $6 \mathrm{HCs}$. Blood was collected after taking signed informed consent. PBMCs were isolated by Ficoll gradient centrifugation and pre-treated with $25 \mu \mathrm{M}$ PNLA or vehicle and then activated with $100 \mathrm{ng} / \mathrm{m}$ LPS. RNA was extracted followed by library construction and sequencing for whole genomic transcriptome. DEGs were analysed using DESeq2 and pathway analysis was performed using Ingenuity pathway analysis. The study was approved by the Research Ethics Committee for Wales (reference no. 12/WA/0045).

Results: DEGs were selected with at least differential genes using at least $1.2^{\star}$ fold changes and adjusted $p$-value of $<0.05$. PNLA significantly upregulates the expression of PDK4, PAI-1 (SERPINE1), FBP1, and NDRG2. These genes have important roles in metabolic pathways. Pathway analysis predicted upstream activation of nuclear receptors peroxisome proliferator-activated receptor (PPARs) involved in the anti-inflammatory process, and inhibition of NF-kB and STAT1, the major transcription factors of the pro-inflammatory cytokines TNF- $\alpha$, IL-1, IL-6 and IFN- $\gamma$.

Conclusion: PNLA has significant effects on the regulation of metabolic and inflammatory pathways in PBMCs from RA patients and HCs. Based on these results we demonstrate that PNLA may have beneficial anti-inflammatory effects in patients with RA

\section{REFERENCES:}

[1] Agca R, Heslinga SC, Rollefstad S, Heslinga M, McInnes. IB, Peters MJL, Kvien, TK., Dougados, M, Radner, H, Atzeni, F. and Primdahl, J. 2017. EULAR recommendations for cardiovascular disease risk management in patients with rheumatoid arthritis and other forms of inflammatory joint disorders: 2015/2016 update. Annals of the rheumatic diseases, 76(1), pp.17-28.

[2] Smolen JS, Landewé RBM, Bijlsma JWJ, et al. 2019. EULAR recommendations for the management of rheumatoid arthritis with synthetic and biological disease-modifying antirheumatic drugs: updateAnnals of the Rheumatic Diseases 2020; 79:685-699. 
[3] Chehade L, Jaafar ZA, El Masri D, Zmerly H, Kreidieh D, Tannir H, Itani L, and El Ghoch, M. 2019. Lifestyle Modification in Rheumatoid Arthritis: Dietary and Physical Activity Recommendations Based on Evidence. Current rheumatology reviews, 15(3), pp.209-214.

[4] Marchand NE, Chiu Y-H, Yoshida K, Malspeis S, Sparks JA, Costenbader K, et al. Threshold Level for Long-term Healthy Diet Adherence to Reduce the Risk of Rheumatoid Arthritis Among Women in a Prospective Cohort Using a Marginal Structural Model Approach.:25

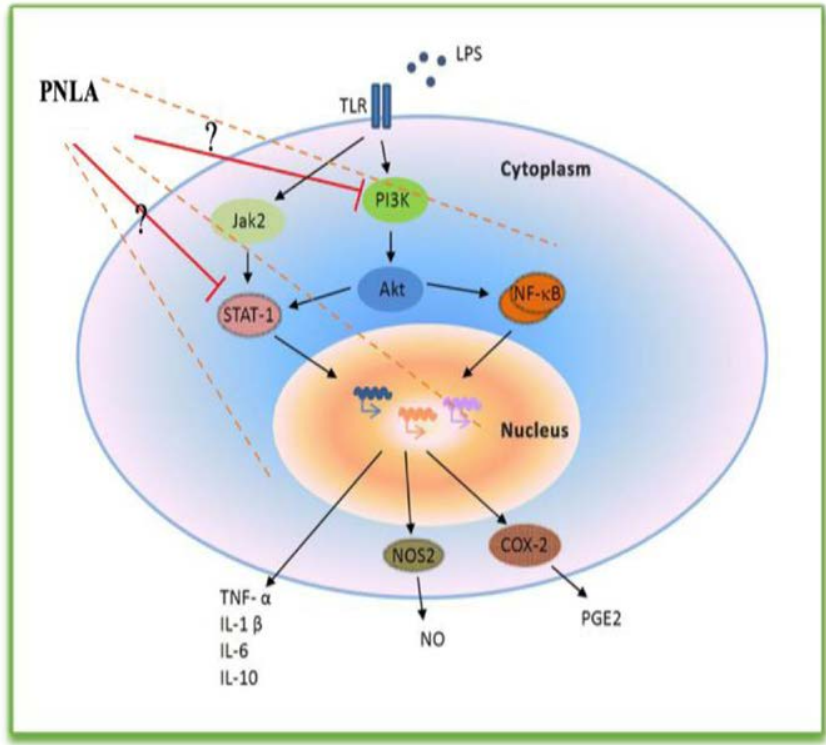

Diagram 1.

Disclosure of Interests: None declared

DOI: 10.1136/annrheumdis-2021-eular.747

\section{POS0347 SPECIFIC MITO-NUCLEAR INTERACTIONS AND MT16519C VARIANT AS PREDICTIVE BIOMARKERS FOR THE RAPIDLY PROGRESSIVE OSTEOARTHRITIS OF THE KNEE. DATA FROM THE OSTEOARTHRITIS INITIATIVE}

A. Durán-Sotuela ${ }^{1}$, M. Fernandez-Moreno ${ }^{1}$, M. E. Vazquez Mosquera ${ }^{1}$, P. RamosLouro' ${ }^{1}$, A. Dalmao-Fernandez ${ }^{1}$, S. Relaño-Fernandez ${ }^{1}$, V. Suárez-Ulloa ${ }^{2}$, V. Balboa-Barreiro ${ }^{3}$, N. Oreiro ${ }^{1}$, J. Vázquez García ${ }^{1}$, F. J. Blanco ${ }^{1}$, I. RegoPerez ${ }^{1}$ on behalf of Grupo de investigación en Reumatología (GIR). ${ }^{1}$ Instituto de Investigación Biomédica de A Coruña (INIBIC). Complexo Hospitalario Universitario de A Coruña (CHUAC), Sergas. Universidade da Coruña., Servicio de Reumatología, A Coruña, Spain; ${ }^{2}$ Instituto de Investigación Biomédica de A Coruña (INIBIC). Complexo Hospitalario Universitario de A Coruña (CHUAC), Sergas. Universidade da Coruña., Plataforma de Bioinformática, A Coruña, Spain: ${ }^{3}$ Instituto de Investigación Biomédica de A Coruña (INIBIC). Complexo Hospitalario Universitario de A Coruña (CHUAC), Sergas. Universidade da Coruña., Unidad de apoyo a la investigación, A Coruña, Spain

Background: The early identification of patients with rapid progressive osteoarthritis (RPOA) could allow the implementation of prevention strategies and their inclusion in clinical trials. Polymorphisms in nuclear and mitochondrial DNA (mtDNA) have been associated with OA. Preliminary analyses by our group showed nuclear single nucleotide polymorphism (nSNP) rs12107036 of TP63 as a potential risk factor for RPOA of the knee.

Objectives: i) To analyze interactions between mtDNA haplogroups and rs12107036 ii) To apply Next Generation Sequencing (NGS) to discover novel mitochondrial variants to construct predictive models of RPOA of the knee.

Methods: 1102 Caucasian subjects from the OAI were classified as follows: i) Rapid progressors $(\mathrm{N}=255)$, baseline $\mathrm{KL}$ grade $0-1$ or 2 in at least one knee, that increases up to $K L \geq 3$ or 4 respectively during 48 -month follow-up. ii) Non-rapid progressors $(\mathrm{N}=847)$, with the same baseline characteristics as rapid progressors, but with slower or no evolution over time.

mtDNA haplogroups and rs 12107036 were assigned by mini-sequencing techniques. Novel mtDNA variants were studied by NGS. Statistical analyses included chi-square tests and generalized estimating equations. Relative excess risk due to interaction (RERI) and attributable proportion (AP) were evaluated for the additive interaction between mtDNA clusters and nSNP rs12107036. A nomogram for the estimation of the risk of RPOA was also developed. Analyses were performed using SPSS Statistics v24 and epi.R package included in R software v3.6.3.
Results: Chi-square analyses revealed an increased risk of RPOA in patients with the allele $\mathrm{G}$ of rs12107036 and mtDNA cluster UK (OR 2,013; $p=0,001$ ). An excess of $70,3 \%$ of RERI between nSNP rs 12107036 and mtDNA clusters was detected, indicating that $47,1 \%$ (AP) of the risk is attributable to this interaction, therefore harboring both genetic factors increase the risk of RPOA up to 4,7 times compared to harboring just one. mtDNA sequencing revealed the variant $m t 16519$ overrepresented in rapid-progressors (OR 1,620; $p=0,002$ ).

Table 1 shows the predictive model for the risk of RPOA. The interaction between the allele $\mathrm{G}$ of rs 12107036 and mtDNA cluster KU (OR 1,727; $p=0,036)$, in addition to the variant $\mathrm{mt} 16519 \mathrm{C}$ (OR 1,690; $p=0,003$ ), showed a significant association with the RPOA phenotype regardless of age, BMI, contralateral knee OA, previous injury and WOMAC pain. Image 1 displays the nomogram for predicting risk of RPOA; as an example, a 70 year old male, with a BMI of 28 , WOMAC pain score of 10 , contralateral OA and presence of both mito-nuclear interaction and $\mathrm{mt} 16519 \mathrm{C}$, has a risk of RPOA of 0,7 .

Conclusion: mtDNA genetic variants are useful, not only as modulators of the influence of specific nuclear polymorphisms on the risk of developing RPOA, but also as candidate genetic biomarkers of this phenotype.

Table 1. Predictive model for the risk of RPOA phenotype

\begin{tabular}{|c|c|c|c|c|}
\hline Variable & p-value & OR & $\min 95 \% \mathrm{Cl}$ & MAX $95 \% \mathrm{Cl}$ \\
\hline \multicolumn{5}{|c|}{ Clinical and genetic variables } \\
\hline Age & $<0,001^{\#}$ & 1,056 & 1,038 & 1,074 \\
\hline Female & 0,143 & 1,260 & 0,925 & 1,718 \\
\hline BMI & $<0,001^{\#}$ & 1,065 & 1,030 & 1,101 \\
\hline Contralateral OA & $<0,001^{\#}$ & 1,927 & 1,413 & 2,626 \\
\hline Previous Injury & $<0,001^{\#}$ & 1,770 & 1,293 & 2,422 \\
\hline WOMAC pain & $0,001^{\#}$ & 1,097 & 1,039 & 1,159 \\
\hline rs $12107036 \mathrm{G}$ & 0,172 & 1,226 & 0,915 & 1,643 \\
\hline \multicolumn{4}{|l|}{ mtDNA Clusters ${ }^{s}$} & 2,375 \\
\hline Others & 0,803 & 0,921 & 0,482 & 1,760 \\
\hline TJ & 0,482 & 1,209 & 0,712 & 2,052 \\
\hline UK & 0,136 & 0,698 & 0,435 & 1,120 \\
\hline HV & & & & Reference \\
\hline \multicolumn{5}{|c|}{ rs12107036 G*mtDNA Cluster } \\
\hline$G$ * Others & 0,502 & 0,789 & 0,395 & 1,576 \\
\hline$G * T J$ & 0,158 & 0,647 & 0,353 & 1,185 \\
\hline$G * U K$ & $0,036^{\#}$ & 1,727 & 1,036 & 2,881 \\
\hline $\mathrm{G} * \mathrm{HV}$ & \multicolumn{4}{|c|}{ Reference } \\
\hline
\end{tabular}

mtDNA Clusters: haplogroups with a common phylogenetic origin BMI: Body Mass Index WOMAC: Western Ontario and McMaster Universities Osteoarthritis Index; OR: Odds Ratio; $\mathrm{Cl}$ : confidence interval; \#: statistical significance declared at $\mathrm{P} \leq 0.05$, in bold.

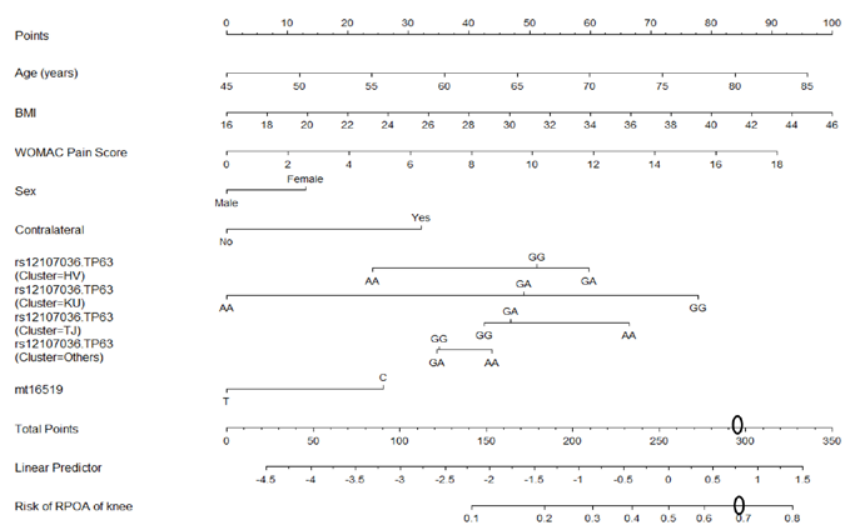

Image 1. Nomogran for the estimation of the risk of RPOA phenotype. Circles represent the values for the example. Clusters: haplogroups with a common phylogenetic origin BMI: Body Mass Index; WOMAC: Western Ontario and McMaster Universities Osteoarthritis Index.

Disclosure of Interests: None declared

DOI: 10.1136/annrheumdis-2021-eular.750

\section{POS0348 GENETIC SUSCEPTIBILITY VARIANTS FOR RHEUMATOID ARTHRITIS ARE NOT ASSOCIATED WITH EARLY REMISSION; A MULTI-COHORT STUDY}

S. Jurado Zapata ${ }^{1}$, M. Maurits ${ }^{1}$, Y. Abraham² ${ }^{2}$ E. Van den Akker ${ }^{3}$, A. Barton ${ }^{4}$, P. Brown ${ }^{5}$, A. Cope $^{6}$, I. González-Álvaro ${ }^{7}$, C. Goodyear ${ }^{8}$, A. van der Helm - van Mil ${ }^{1}$, X. $\mathrm{Hu}^{9}$, T. Huizinga ${ }^{1}$, M. Johannesson ${ }^{10}$, L. Klareskog ${ }^{10}$, D. Lendrem ${ }^{11}$, I. Mclnnes ${ }^{12}$ F. Morton ${ }^{12}$, C. Paterson ${ }^{12}$, D. Porter ${ }^{12}$, A. Pratt ${ }^{11}$, L. Rodriguez Rodriguez ${ }^{13}$,

D. Sieghart ${ }^{14}$, P. Studenic ${ }^{14}$, S. Verstappen ${ }^{4}$, L. Padyukov ${ }^{10}$, A. Winkler ${ }^{9}$, J. D. Isaacs ${ }^{5}$, 